\title{
Poisson's Ratio and the Densification of Glass under High Pressure
}

\author{
T. Rouxel, ${ }^{1}$ H. Ji, ${ }^{1}$ T. Hammouda, ${ }^{2}$ and A. Moréac ${ }^{3}$ \\ ${ }^{1}$ Applied Mechanics Laboratory of the University of Rennes 1, LARMAUR, Université de Rennes 1, \\ Campus de Beaulieu, 35042 Rennes cedex, France \\ ${ }^{2}$ Laboratoire Magmas et Volcans, CNRS-OPG, Université Blaise Pascal, 5 rue Kessler, 63038 Clermont-Ferrand cedex, France \\ ${ }^{3}$ IPR, CNRS-Université de Rennes 1, Campus de Beaulieu, 35042 Rennes cedex, France
}

(Received 3 January 2008; published 3 June 2008)

\begin{abstract}
Because of a relatively low atomic packing density, $\left(C_{g}\right)$ glasses experience significant densification under high hydrostatic pressure. Poisson's ratio $(\nu)$ is correlated to $C_{g}$ and typically varies from 0.15 for glasses with low $C_{g}$ such as amorphous silica to 0.38 for close-packed atomic networks such as in bulk metallic glasses. Pressure experiments were conducted up to $25 \mathrm{GPa}$ at $293 \mathrm{~K}$ on silica, soda-lime-silica, chalcogenide, and bulk metallic glasses. We show from these high-pressure data that there is a direct correlation between $\nu$ and the maximum post-decompression density change.
\end{abstract}

DOI: 10.1103/PhysRevLett.100.225501

PACS numbers: 61.43.Fs, 62.20.- $\mathrm{x}, 81.05 . \mathrm{Kf}$

Introduction. - It is well known that the density of glasses can be increased when a pressure up to $25 \mathrm{GPa}$ is applied [1-3]. Hydrostatic stresses of the same order of magnitude develop in glasses under sharp contact loading, such as Vickers hardness (hardness which is a mean value of the contact pressure is typically of few GPa) and nanoindentation testing. Therefore high-pressure experiments are of paramount interest to get insight into the mechanism of the inelastic indentation deformation observed in glasses at room temperature. In particular, such investigations are required to estimate the densification contribution to the indentation formation with respect to volume conservative shear flow. It was previously showed that densification plays a major role in amorphous silica whereas volume conservative shear flow predominates in bulk metallic glasses [4]. However, so far high-pressure investigations mostly focused on the subtle structural changes occurring at the atomic scale and were limited to very few glass systems among which silica is by far the most documented one. Data on the post-decompression density change are especially scarce, either because specimens fragmented during the test-which means that the loading was not perfectly hydrostatic - or because the specimens were too small. Previous studies showed that in the case of $\mathrm{SiO}_{2}$ glass, the density can be increased by more than $15 \%$ and reaches the one of quartz when a pressure higher than $15 \mathrm{GPa}$ is applied [2,3].

Poisson's ratio is the negative of the ratio of transverse contraction strain to longitudinal extension strain in the direction of the elastic loading. Hence, $\nu$ reflects the resistance a material opposes to volume change vis-à-vis shape change. $\nu$ is small for shear-resistant compressible materials, such as cellular solids, but tends toward 0.5 for incompressible bodies such as rubber. Glasses exhibit a wide range of values for $\nu$ from 0.1 to 0.4 . It was shown first in the case of oxide glasses [5] and recently for a broader range of glass compositions [6] that $\nu$ is directly correlated to the atomic packing density $\left(C_{g}\right) . C_{g}$ is defined as the ratio between the minimum theoretical volume occupied by the atoms and the corresponding effective volume of glass. Maximum values for $\nu$ and $C_{g}$ are observed for metallic glasses $\left(\nu \sim 0.4\right.$ and $\left.C_{g}>0.7\right)$ whereas silica-rich glasses exhibit the lowest values $(\nu<0.2$ and $C_{g}<0.5$ ). Hence, Poisson's ratio-which can be easily measured by ultrasonic echography for instance-shows up as an interesting parameter reflecting the free volume fraction in glasses which undoubtedly affect the high pressure-densification behavior.

Pressure-densification data were obtained on a $\mathrm{Zr}_{55} \mathrm{Cu}_{30} \mathrm{Al}_{10} \mathrm{Ni}_{5}$ bulk metallic glass, a $\mathrm{GeSe}_{4}$ chalcogenide glass, and a silica glass (vitreosil, Saint-Gobain, Fr) by means of an octahedral multianvil apparatus using a Walker cell and following a procedure described elsewhere $[7,8]$. The high-pressure cell consists of a $\mathrm{Cr}$-doped $\mathrm{MgO}$ octahedron which is squeezed between eight converging truncated cubic tungsten carbide anvils. Starting glass cylinders were wrapped in $25 \mu \mathrm{m}$ thick gold foils in order to prevent contact with the ceramic parts of the highpressure assemblies. It is noteworthy that unlike previously reported experiments, most specimens came out in one piece, suggesting that the pressure device induced very little shear. Besides, the treatment proved to be very homogeneous as demonstrated by preliminary micro-Raman scattering investigations along the specimen axis. Density was measured with a better than $0.001 \mathrm{~g} \cdot \mathrm{cm}^{-3}$ accuracy either by means of a density gradient method using two partially miscible liquids (iodobenzene and methylen iodide were used for silica) or by means of image analysis, using high resolution images of the specimens taken prior to-and after-testing. An excellent agreement was found between both techniques. Pressure-densification data are plotted together with previously reported data on glassy $\mathrm{SiO}_{2}$ [2] and $\mathrm{B}_{2} \mathrm{O}_{3}$ [1], on a $\mathrm{F}_{57} \mathrm{Ba}_{15} \mathrm{Eu}_{5} \mathrm{Zr}_{3}$ [9] fluoride glass, and on a standard soda-lime-silica window glass (WG) [10] in Fig. 1. The maximum observed relative density change was associated with the apparent saturation 


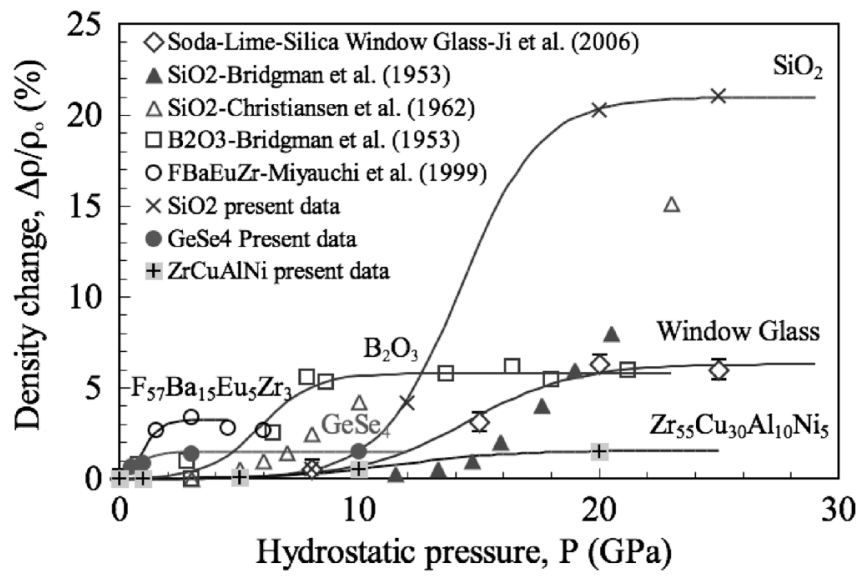

FIG. 1. Change of the relative density as a function of the applied pressure at room temperature (post-decompression measurements).

of the densification. Although Bridgman did not see any saturation in the case of $a-\mathrm{SiO}_{2}$, Brillouin scattering experiments [11] reveal a density plateau at $\sim 25 \mathrm{GPa}$. Our data confirm this study and give a maximum density change of $21 \%$ that is significantly larger than the maximum change $(\sim 15 \%)$ reported in earlier studies [1,2]. Furthermore, we observe an onset for densification at $\sim 10 \mathrm{GPa}$ in excellent agreement with the rise of the frequency shift observed by Brillouin scattering. In the cases of $\mathrm{GeSe}_{4}, \mathrm{GeO}_{2}[3,12,13]$, and $\mathrm{B}_{2} \mathrm{O}_{3}$, there are no observable threshold pressures. Data were smoothly fitted with a sigmoid function,

$$
\frac{\Delta \rho}{\rho}=\frac{\alpha}{1+\beta \exp \left(-P / P_{0}\right)}-\frac{\alpha}{1+\beta},
$$

where $\Delta \rho / \rho(\%)$ is the fraction of inelastic density change, $P$ is the pressure (in GPa), and $\left(\alpha, \beta, P_{0}\right)$ are curve fitting parameters $(\alpha$ is the maximum relative density change at the apparent saturation plateau). $\left(\alpha, \beta, P_{0}\right)$ values of (21, 5000, 1.67), (5.8, 200, 1.1), (2, 3, 0.48), (6.3, 700, 2.22), $(1.6,100,2.5)$ and $(3.4,20,0.33)$ were estimated for $\mathrm{SiO}_{2}$, $\mathrm{B}_{2} \mathrm{O}_{3}, \mathrm{GeSe}_{4}, \mathrm{WG}, \mathrm{Zr}_{55} \mathrm{Cu}_{30} \mathrm{Al}_{10} \mathrm{Ni}_{5}$, and $\mathrm{F}_{57} \mathrm{Ba}_{15} \mathrm{Eu}_{5} \mathrm{Zr}_{3}$ glasses, respectively.

The structural changes induced by the high-pressure experiments in amorphous silica, window glass, and chalcogenide glass were investigated post-decompression by Raman scattering spectroscopy (Fig. 2). The broadness of the bands still indicates amorphous phases. However, it is generally difficult to attach a clear significance to the slight shifts observed on the position of the maxima for the pressure-densified glass specimens. In the case of amorphous silica [Fig. 2(a)], the sharpening of the main band near $500 \mathrm{~cm}^{-1}$ and its shift to higher frequency $\left(427 \mathrm{~cm}^{-1}\right.$ in the pristine glass and $511 \mathrm{~cm}^{-1}$ after densification under $25 \mathrm{GPa}$ ) reveals a narrower distribution and a decrease of the Si-O-Si intertetrahedral angle, consistently with earlier investigations interpreted on the basis of a central-forcemodel [14] and with the prediction of molecular-dynamics
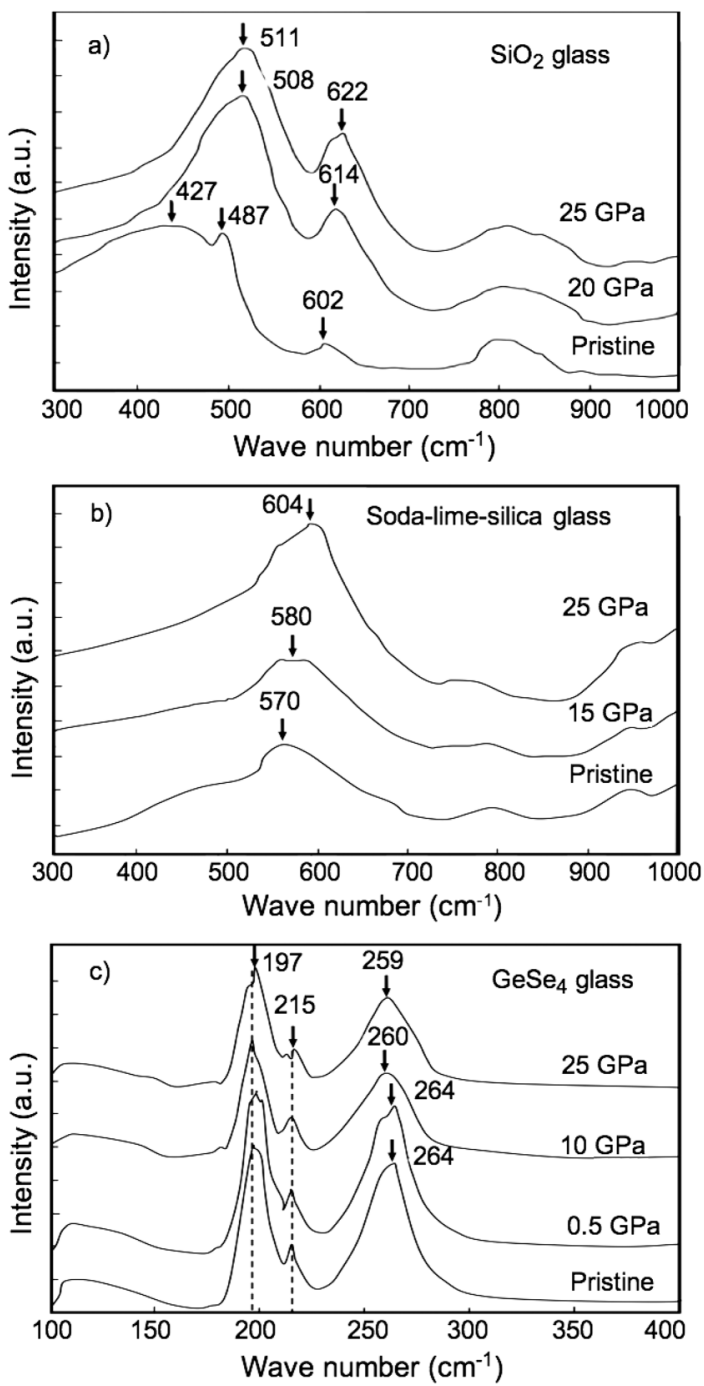

FIG. 2. Room temperature Raman spectra of the glass specimens before (pristine) and after high-pressure treatments: (a) Amorphous silica; (b) Window glass; and (c) $\mathrm{GeSe}_{4}$.

simulation [15]. The main band shifts to higher frequency as pressure is increased and seems to overlap the shoulder band at $\sim 487$ (also known as D1 band). There is also an increase and a shift to higher frequency of the relatively sharp peak at $602 \mathrm{~cm}^{-1}$ (also known as D2 band). D1 and D2 arise from symmetric oxygen atoms breathing motion of small rings consisting of 4 (D1) and 3 (D2) $\mathrm{SiO}_{4}$ tetrahedra respectively $[16,17]$. The shift of D2 from 602 to $622 \mathrm{~cm}^{-1}$ for a density increase from 2.2 to $2.66 \mathrm{~g} \cdot \mathrm{cm}^{-3}$ agrees well with the extrapolation of the $\mathrm{D} 2$ position versus density curve reported earlier [18]. It is noteworthy that the rather broad band at about $810 \mathrm{~cm}^{-1}$ and ascribed to vibrational mode of 6-membered rings of $\mathrm{SiO}_{4}$ units [19] becomes more symmetrical after the densification process. In the case of window glass, the densification is much less pronounced than in amorphous silica, and the analysis of the light changes in the Raman spectra is difficult. Nevertheless, high-pressure treatments lead to a shift of the main band located at $570 \mathrm{~cm}^{-1}$ to higher frequency 
(604 $\mathrm{cm}^{-1}$ after densification under $25 \mathrm{GPa}$ ) as a result of the decrease of the mean intertetrahedral angle, consistently with a former study on soda-alumina-silica glasses [16]. It is noteworthy that the increase of the frequency at $\sim 570 \mathrm{~cm}^{-1}$ can also be interpreted in the light of the changes observed on $\mathrm{Na}_{2} \mathrm{O}-\mathrm{SiO}_{2}$ silicates [20]. In the former case, a shift of this band to higher frequency was noticed when the number of bridging oxygen atoms $\left(n_{\mathrm{BO}}\right)$ per tetrahedron decreased. For a window glass ( $71 \mathrm{~mol}^{\circ} \% \mathrm{SiO}_{2}, 13 \mathrm{~mol} . \% \mathrm{Na}_{2} \mathrm{O}$ and $16 \mathrm{~mol} . \% \mathrm{CaO}+$ $\mathrm{MgO}$ ), the frequency shift would correspond to a drop of $n_{\mathrm{BO}}$ from 3.18 to 2.36 , i.e., a change from pyrosilicate units (sheetlike symmetry) to metasilicate (chainlike units).

The occurrence of low symmetry structural units was predicted by molecular dynamic simulation in $\mathrm{GeSe}_{2}$ glass [21], with the emergence of chainlike Se-Se clusters at high pressure, whereas a 3D organization allowing for $\mathrm{Ge}-$ Se bonds only is predicted from the glass stoichiometry. Moreover, in the former case, the compressibility is governed by the decrease of the intertetrahedral Ge-Se-Ge bond angle leaving Ge-Se bond length almost constant and pressure-independent [22]. Again, pressure seemed to mainly affect the intermediate range order with little incidence on the short range characteristics. The results of the high-pressure experiments on the $\mathrm{GeSe}_{4}$ composition tie up well with high-pressure electrical resistivity experiments [23]. Both electrical conductivity and density increase with rising pressure, but changes become very limited beyond $8 \mathrm{GPa}$. The Raman spectrum of the pristine glass [Fig. 2(c)] reveals three bands located at 197, 215, and $260 \mathrm{~cm}^{-1}$ and assigned to the symmetric breathing vibration of Se atoms at corner-sharing $\mathrm{GeSe}_{4 / 2}$ tetrahedra, to the breathing vibration of edge-sharing bitetrahedra, and to the Se-Se stretching mode of $(-\mathrm{Se}-)_{n}$ chains respectively $[24,25]$. These structural arrangements are not expected from the glass stoichiometry. Indeed, on the basis of a random atomic organization, a $\mathrm{GeSe}_{4}$ glass should consist of $\mathrm{GeSe}_{4 / 2}$ tetrahedra connected together by Se-Se bridges, as depicted by the schematic drawing inserted in Fig. 3. The observed discrepancy supports the clustering model [26] which assumes the coexistence of $(-\mathrm{Se}-)_{n}$ chains (or rings) and Ge-Se-Ge bonds even for Se content below $20 \%$.

The main incidence of the high-pressure treatments are the decrease and the shift to lower frequency of the band at $\sim 260 \mathrm{~cm}^{-1}$ and a slight decrease of the shoulder of the main band at $215 \mathrm{~cm}^{-1}$ which can be partially attributed to the shift of the main band at $197 \mathrm{~cm}^{-1}$ to higher frequency. These observations suggest that pressure induces an increase of the Se-Se bond length and a decrease of the fraction of edge-sharing tetrahedra, probably to the benefit of corner-sharing ones. Although the structure of the densified bulk metallic specimens was not characterized, previous investigations [27] showed that these glasses which have dense packed atomic configurations experience rather small structural changes, most of the volumetric contraction being recovered after the pressure is relieved. Our data

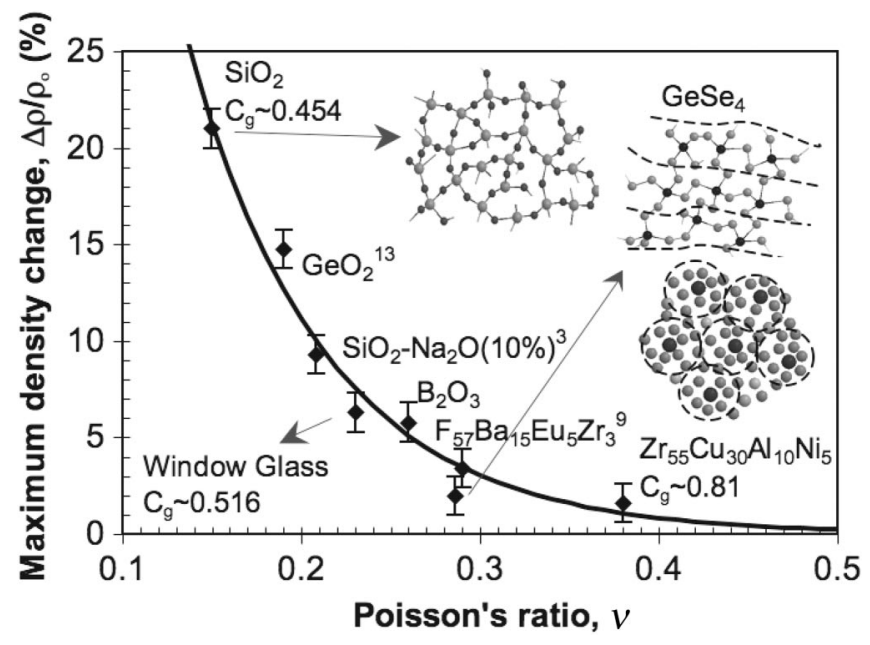

FIG. 3. Maximum observed changes of the relative specific mass (apparent saturation plateau) after high-pressure treatments at room temperature as a function of Poisson's ratio, as calculated from the velocities of transverse $\left(V_{t}\right)$ and longitudinal $\left(V_{l}\right)$ waves measured by means of ultrasonic echography for $\mathrm{SiO}_{2}$, WG, $\mathrm{GeSe}_{4}$, and $\mathrm{Zr}_{55} \mathrm{Cu}_{30} \mathrm{Al}_{10} \mathrm{Ni}_{5}$ glasses and taken from Ref. [6] for the others. Recall that $\nu=\left(V_{l}^{2}-2 V_{t}^{2}\right) /\left[2\left(V_{1}^{2}-\right.\right.$ $\left.\left.V_{t}^{2}\right)\right]$.

on $\mathrm{Zr}_{55} \mathrm{Cu}_{30} \mathrm{Al}_{10} \mathrm{Ni}_{5}$ composition suggest a maximum density change close to $2 \%$.

Generally speaking, the densification process is associated with a significant decrease of the intertetrahedral angle (Ge-Se-Ge and $\mathrm{Si}-\mathrm{O}-\mathrm{Si}$ angles for instance), small or undetectable changes of the interatomic distances (adjacent neighbors), and a gradual increase of the average atomic coordination (from 4 to 6 in amorphous silica, from 3 to 4 in $\mathrm{B}_{2} \mathrm{O}_{3}$ glass, from fourfold to sixfold for $\mathrm{Ge}$ and from twofold to fourfold for $\mathrm{Se}$ in $\mathrm{Ge}_{x} \mathrm{Se}_{1-x}$ glasses).

Furthermore, at room temperature at least, it seems that although pressure causes a slight reduction of the chemical disorder (bond angles and bond lengths distributions become narrower), it leads to a greater topological disorder at the medium range scale. As a matter of fact, Poisson's ratio, which is below 0.2 for highly polymerized glass atomic networks and approaches 0.4 for glass networks consisting of chain and cluster units, is expected to evolve during high-pressure treatments and to correlate with the magnitude of the densification process. In the case of amorphous silica [28], an increase of $\nu$ from 0.15 to 0.33 was already evidenced after a pressure cycle up to $25 \mathrm{GPa}$. The correlation between $\nu$ and the magnitude of the density increase is the major finding of this study and is discussed below.

Although Poisson's ratio is strictly defined only for small strain linear elastic behavior, a remarkable outcome of the present experiments is that there is an excellent correlation between this ratio and the magnitude of the densification process (Fig. 3) which involves large strain. We think that this correlation stems from the fact that $\nu$ depends much on the glass atomic network organization. 
For instance, relatively open structures such as observed in amorphous silica and germania usually come with a high polymerization degree (silica and germania glasses are based on corner-sharing tetrahedra) so that a strong resistance toward contraction in transverse direction shows up in tension, and consequently the glass exhibits a low Poisson's ratio ( 0.15 and 0.19 , respectively). When the atomic cross-linking degree decreases, the atomic packing increases (it is easier to pack rod or sheetlike units than tetrahedra), but local shear becomes easier so that Poisson's ratio increases.

For instance, $\mathrm{B}_{2} \mathrm{O}_{3}$ glass - consisting of corner-sharing planar triangular $\mathrm{BO}_{3}$ units which are weakly bonded together (unlike crystallized $\mathrm{B}_{2} \mathrm{O}_{3}$ consisting of a tridimensional network) — can be considered as possessing a dimensionality lower than the one of silica and is characterized by Poisson's ratio of 0.26 . The same holds for $\mathrm{GeSe}_{4}$ glass consisting of a complex mixtures of $1 \mathrm{D}(-\mathrm{Se}-)_{n}$ segments and 3D GeSe $\mathrm{S}_{4}$ tetrahedra - for which $\nu=0.286$. On the contrary to amorphous silica, the $\mathrm{Zr}_{55} \mathrm{Cu}_{30} \mathrm{Al}_{10} \mathrm{Ni}_{5}$ bulk metallic glass exhibits a rather close-packed atomic network $\left(C_{g} \sim 0.8\right)$ and hence a very high Poisson's ratio $(\nu \sim 0.38)$ among inorganic glasses. Recalling that $\nu$ approaches 0.5 for liquids - perfect incompressibility corresponds to $\nu=0.5$-it can be anticipated that an increase of Poisson's ratio will correspond to a smaller extent of the densification process. This result is corroborated by a recent theoretical study of glasses from the $(\mathrm{Ge}, \mathrm{Se})$ chemical system and concluding that densification is favored in glasses with high average coordination number [29] which also turn to be those with small Poisson's ratio [6]. According to the present study, $\nu$ can be viewed as a useful index to predict the extent of densification of a glass with an arbitrary composition. The experimental data in Fig. 3 were nicely fitted with the simple following relationship:

$$
\frac{\Delta \rho}{\rho_{o}}(\%)=A \exp (-B v) \quad(A \approx 150 \text { and } B \approx 13) .
$$

Hence, $\nu$ does not only allow for the estimation of the volume change in a small-scale elastic deformation regime [ $\Delta V / V$ is proportional to $(1-2 \nu)]$, but it seems to allow for the prediction of the achievable densification of glass at ambient temperature for pressure up to $25 \mathrm{GPa}$. Moreover, our data suggest that the pressure corresponding to the onset of the densification process is linked to the bulk elastic modulus $(K$ reaches 113.1, 33.3, 23.3, 12.1, and 11.5 GPa for $\mathrm{Zr}_{55} \mathrm{Cu}_{30} \mathrm{Al}_{10} \mathrm{Ni}_{5}$, silica, germania, $\mathrm{B}_{2} \mathrm{O}_{3}$ and $\mathrm{GeSe}_{4}$ glasses, respectively). Glasses with low $K$ values such as $\mathrm{B}_{2} \mathrm{O}_{3}$ and $\mathrm{GeSe}_{4}$ experience inelastic densification at pressure as low as $1 \mathrm{GPa}$ whereas there seems to exist a threshold pressure between 8 and $10 \mathrm{GPa}$ for $a-\mathrm{SiO}_{2}$ as well as for the window and the metallic glasses.

In conclusion, this Letter shows that the sensitivity of glasses from very different chemical systems to density changes under high pressure depends much on their chemical composition and is linked to the atomic packing den- sity. Our main finding is that Poisson's ratio which covers a wide interval of values for inorganic glasses, is correlated to the maximum density improvement. The smaller $\nu$ is and the larger the density improvement becomes. Therefore, $\nu$ is proposed as a simple estimator of the density change observed in a glass of arbitrary composition after high-pressure experiments.

Funding for this project was provided by the Ministry of Research and Higher Education (Grant No. HJ1308761207).

[1] P. W. Bridgman and I. Simon, J. Appl. Phys. 24, 405 (1953).

[2] E. B. Christiansen, S. S. Kistler and W. B. Gogarty, J. Am. Ceram. Soc. 45, 172 (1962).

[3] J. D. Mackenzie, J. Am. Ceram. Soc. 46, 461 (1963).

[4] S. Yoshida, J.-C. Sangleboeuf and T. Rouxel, J. Mater. Res. 20, 3404 (2005).

[5] A. Makishima and J. D. Mackenzie, J. Non-Cryst. Solids 17, 147 (1975).

[6] T. Rouxel, J. Am. Ceram. Soc. 90, 3019 (2007).

[7] D. Walker, M. A. Carpenter, and C. M. Hitch, Am. Mineral. 75, 1020 (1990).

[8] T. Hammouda, Earth Planet. Sci. Lett. 214, 357 (2003).

[9] K. Miyauchi et al., Mater. Res. Bull. 34, 1383 (1999).

[10] H. Ji, V. Keryvin, T. Rouxel, and T. Hammouda, Scr. Mater. 55, 1159 (2006).

[11] M. Grimsditch, Phys. Rev. Lett. 52, 2379 (1984).

[12] H. M. Cohen and R. Roy, J. Am. Ceram. Soc. 44, 523 (1961).

[13] P. Richet, G. Hovis, and B. Poe, Chem. Geol. 213, 41 (2004).

[14] R. J. Hemley et al., Phys. Rev. Lett. 57, 747 (1986).

[15] J.S. Tse, D. Kug, and Y. Le Page, Phys. Rev. B 46, 5933 (1992).

[16] B. Mysen et al., Neues Jahrbuch fur MineralogieAbhandlungen 147, 281 (1983).

[17] F. L. Galeener et al., Phys. Rev. Lett. 53, 2429 (1984).

[18] H. Sugiura and T. Yamadaya, J. Non-Cryst. Solids 144, 151 (1992).

[19] F. Seifert, B. O. Mysen, and D. Virgo, Am. Mineral. 67, 696 (1982).

[20] T. Furukawa, K. E. Fox, and W. B. White, J. Chem. Phys. 75, 3226 (1981).

[21] M. Durandurdu and D. A. Drabold, Phys. Rev. B 65, 104208 (2002).

[22] W. A. Crichton et al., Nature (London) 414, 622 (2001).

[23] S. Asokan et al., Phys. Rev. Lett. 62, 808 (1989).

[24] K. Murase et al., J. Non-Cryst. Solids 59\&60, 883 (1983).

[25] P. Boolchand, X. Feng, and W. J. Bresser, J. Non-Cryst. Solids 293-295, 348 (2001).

[26] B. Bureau et al., J. Non-Cryst. Solids 319, 145 (2003).

[27] L. M. Wang et al., J. Phys. Condens. Matter 13, 5743 (2001).

[28] C. S. Zha et al., Phys. Rev. B 50, 13105 (1994).

[29] J. C. Mauro and A. K. Varshneya, J. Am. Ceram. Soc. 90, 192 (2007). 